\title{
Factors Associated with Chemotherapy Induced Nausea in Cancer Patients: A Case-Control Study
}

\author{
Mahnaz Khatiban (iD) ${ }^{1}$, Zahra Khalili ${ }^{2,{ }^{*}}$, Mahdi Akbarzadeh ${ }^{3}$, Mahnaz Moghadarikoosha ${ }^{4}$ and Maryam \\ Seyedtabib ${ }^{5}$ \\ ${ }^{1}$ Mother \& Child Care Research Center, Department of Ethics Education in Medical Sciences, Department of Medical-Surgical Nursing, School of Nursing and Midwifery, \\ Hamadan University of Medical Sciences, Hamadan, Iran \\ ${ }^{2}$ Chronic Disease (Home Care) Research Center, Department of Medical Surgical Nursing, Faculty of Nursing and Midwifery, Hamadan University of Medical Sciences, \\ Hamadan, Iran \\ ${ }^{3}$ Cellular and Molecular Research Center, Research Institute for Endocrine Sciences, Shahid Beheshti University of Medical Sciences, Tehran, Iran \\ ${ }^{4}$ Department of Operating Room, Faculty of Paramedical, Hamadan University of Medical Sciences, Hamadan, Iran \\ ${ }^{5}$ Department of Biostatistics and Epidemiology, School of Public Health, Ahvaz Jundishapur University of Medical Sciences, Ahvaz, Iran \\ "Corresponding author: Chronic Disease (Home Care) Research Center, Department of Medical Surgical Nursing, Faculty of Nursing and Midwifery, Hamadan University of \\ Medical Sciences, Hamadan, Iran. Email: zkhalili90@yahoo.com
}

Received 2021 March 04; Accepted 2021 April 09.

\begin{abstract}
Background: Chemotherapy-induced nausea (CIN) is one of the most common and uncomfortable symptoms in cancer patients, and different factors can be associated with it.

Objectives: This study aimed to determine different factors associated with CIN in cancer patients.

Methods: A total of 144 cancer patients were selected by convenience sampling. The patients at acute phase of chemotherapy were assigned to case group $(n=58)$ if they had nausea or to control group $(n=86)$ if they did not have nausea. The patients' data were collected using a researcher-made questionnaire including items on potential factors for CIN through interviews with the patients and according to their medical records. Logistic regression models were used to conduct data analysis, and the correlations in question were expressed as odds ratio (OR) at 95\% confidence interval (CI).

Results: The results showed that the chance of nausea increased by 6.4, 2.4, 1.2, and 1.5 times in case of expected nausea, pain, carbohydrate intake, and smelling a specific odor, respectively. The increasing nausea-inducing effect of drugs led to increased chance of post-chemotherapy nausea $(\mathrm{OR}=2.366)$.

Conclusions: Having pain, expecting nausea, carbohydrate intake, smelling a certain odor, and high emetogenic potential of chemotherapy are effective in the development of CIN.
\end{abstract}

Keywords: Chemotherapy, Nausea, Vomiting, Cancer

\section{Background}

Chemotherapy-induced nausea (CIN) is a debilitating side effect of antineoplastic drugs leading to exacerbated disability and declined adherence to the treatment $(1,2)$. Nausea is considered as one of the main side effects due to chemotherapy (3). Chemotherapy-induced nausea and vomiting (CINV) may occur up to 120 hours (five days) after initiation of treatment with antineoplastic drugs and can be acute or late. The acute phase occurs within 24 hours after initiation of chemotherapy, and the late phase occurs within 24 - 120 hours (4). Nausea and vomiting occur mainly within the acute phase, adversely affecting the patients' personal and working life $(5,6)$.

To date, several studies have confirmed the association between certain factors and CIN; these factors include age
(7-10), family support (11), lack of food intake (12), type of chemotherapy drug with different emetogenic potential $(10,13)$, treatment course, and adjuvant radiotherapy (13). However, the association of some factors with CIN is still controversial. While the effects of expecting nausea $(7,14)$ and female gender $(10,13)$ on CIN have been confirmed in some studies, some other studies did not report such an association $(7,8)$. This inconsistency in the findings necessitates further investigation in this regard.

Although, several associated factors have been studied for nausea and vomiting of pregnancy (NVP), and postoperative nausea and vomiting (PONV), these factors were not investigated in the CIN. In this regard, the associations between certain factors such as education level (15), being employed (16), development of nausea after smelling a cer- 
tain odor, and the satisfaction of social support (17) with NVP were studied. In addition, the effects of body mass in$\operatorname{dex}(\mathrm{BMI})(18,19)$, smoking (20), narcotics (21), and pain (22) have been investigated for PONV. As far as the researchers investigated, there is no study to evaluate the correlation between CIN and these factors.

\section{Objectives}

This study aimed to investigate the factors potentially associated with CIN.

\section{Methods}

A case-control study was conducted in the Hematology-Oncology Ward and the Outpatient Chemotherapy Clinic in Hamedan. After the Ethics Committee of the Hamedan University of Medical Sciences approved the study protocol, 144 cancer patients were selected by convenience sampling. The inclusion criteria were being under chemotherapy, sufficiently conscious and able to speak, and receiving the first dose of the chemotherapy medicines since the initiation of the second cycle onwards within the past 24 hours (acute phase). The patients at acute phase of chemotherapy were assigned to case group $(n=58)$ if they had nausea and to control group $(n=86)$ if they did not have nausea.

The data on the patients, both with and without nausea, were collected by a researcher-made questionnaire developed according to the relevant literature. The questionnaire consisted of items on sociodemographic and clinical characteristics. The sociodemographic characteristics included gender (13), age (7-10), BMI (23), education level (15), employment status (16), urbanization, residential type, marital status, having health insurance, and economic status. Information about the food consumption (12), the type of the last food consumption before the chemotherapy, the time interval between the last food consumption and chemotherapy, history of smoking and drug abuse (20), having expectation of nausea following chemotherapy (7, 14), feeling nausea following some factors, and satisfaction of social support (17) were also considered.

The clinical information investigated the type of chemotherapy medicines for emetogenic potential (10), antiemetic agents taken by the patient at acute phase (8), pain (22), the type of cancer, and the number of chemotherapy periods.

The questionnaires were filled out through interviews with the patients and their medical records after completion of the acute phase of chemotherapy.

Data analysis was conducted by SPSS software version 13. Descriptive statistics were expressed as frequency and mean. Chi-square and Fisher's exact tests were used to investigate association between the studied variables and nausea. For all tests, the level of significance was 0.05 . Since the dependent variable was binary, the logistic regression was used to conduct data analysis, and the result was expressed as adjusted odds ratio (OR).

\section{Results}

Most of the participants in the two groups were male, married, literate, unemployed, and lived in urban areas. Most patients in the two groups did not report any history of smoking (Tables 1 and 2).

To investigate the association of the factors between the two groups with CIN, logistic regression models were used according to the crude and adjusted OR.

Logistic regression analysis and backward method were used to evaluate the important factors affecting the nausea according to previous studies. Out of the variables included in the model (type of food, factors affecting nausea, pain in the acute phase, emetogenic potential of chemotherapy drugs, number of chemotherapy periods, BMI, and expecting nausea), several variables, including expected nausea after chemotherapy, pre-chemotherapy carbohydrate consumption, a specific odor, presence of pain in the acute phase of the chemotherapy, and receiving chemotherapy drugs with high nausea-inducing potential were effective on the outcome of post-chemotherapy nausea. Also, the chance of nausea in patients expecting to be at higher risk of post-chemotherapy nausea was 6.458 higher than those who did not have such an expectation. The chance of nausea in patients who consumed carbohydrates before chemotherapy was 1.288 times higher than those who did not eat any food. Smelling a specific smell during the acute phase of chemotherapy led to 1.559-fold increase in the chance of nausea as compared to those who did not smell any odor. Patients who had pain in the acute phase of chemotherapy had a 2.426-fold increase in having the chance of nausea as compared to patients who did not have pain. The increasing rate of nausea-inducing property of the drug led to an increase in the chance of postchemotherapy nausea $(\mathrm{OR}=2.366)$ (Table 3$)$.

\section{Discussion}

In the current study, most subjects, either with or without nausea, had meal before the chemotherapy. There was no significant difference between the two groups in terms of the time interval between eating and starting chemotherapy. The results also showed that the chance of nausea in patients who consumed carbohydrates before 


\begin{tabular}{|c|c|c|c|}
\hline Variables & With Nausea & Without Nausea & P-Value \\
\hline Age (y) & $46.66 \pm 16.98$ & $47.65 \pm 16.67$ & $0.62^{b}$ \\
\hline $\operatorname{BMI}\left(\mathbf{k g} / \mathbf{m}^{2}\right)$ & $24.07 \pm 9.27$ & $22.41 \pm 4.01$ & $0.24^{b}$ \\
\hline Gender & & & $0.447^{\mathrm{c}}$ \\
\hline Male & $30(51.7)$ & $50(58.1)$ & \\
\hline Female & $28(48.3)$ & $36(41.9)$ & \\
\hline Marital status & & & $0.24^{c}$ \\
\hline Married & $40(70.2)$ & $64(74.4)$ & \\
\hline Single & $14(24.6)$ & $13(15.1)$ & \\
\hline Others & $3(5.3)$ & $9(10.4)$ & \\
\hline Urbanization & & & $0.45^{\mathrm{c}}$ \\
\hline Urban & $32(56.1)$ & $53(62.4)$ & \\
\hline Rural & $25(43.9)$ & $32(37.6)$ & \\
\hline Residential type & & & $0.75^{\mathrm{c}}$ \\
\hline Tenant & $11(20)$ & $18(22.2)$ & \\
\hline Landlord & $44(80)$ & $63(77.8)$ & \\
\hline Education level & & & $0.95^{\mathrm{c}}$ \\
\hline Illiterate & $19(33.3)$ & $31(36)$ & \\
\hline Basic education & $24(42.1)$ & $32(37.2)$ & \\
\hline High school diploma & $9(15.8)$ & $15(17.4)$ & \\
\hline Diploma of higher education & $5(8.8)$ & $8(9.3)$ & \\
\hline Employment status & & & $0.81^{\mathrm{c}}$ \\
\hline Employed & $23(41.1)$ & $37(43)$ & \\
\hline Unemployed & $33(59)$ & $49(57)$ & \\
\hline Economic status & & & $0.17^{c}$ \\
\hline Enough & $9(16.4)$ & $8(10)$ & \\
\hline Somewhat enough & $19(34.5)$ & $20(25)$ & \\
\hline Not enough & $27(49.1)$ & $52(65)$ & \\
\hline The time interval between eating and chemotherapy $(\mathrm{h})$ & & & $0.32^{c}$ \\
\hline$<0.5$ & $4(8.3)$ & $10(13.2)$ & \\
\hline $0.5-1$ & $8(16.7)$ & $20(26.3)$ & \\
\hline $1 \cdot 2$ & $8(16.7)$ & $14(18.4)$ & \\
\hline$>2$ & $28(58.3)$ & $32(42.1)$ & \\
\hline Which one affected your nausea in past 24 hours? & & & $0.007^{c}$ \\
\hline Odors & $30(51.7)$ & $27(31.4)$ & \\
\hline Consuming some foods & $6(10.3)$ & $5(5.8)$ & \\
\hline Seeing somethings & $0(0)$ & $3(3.5)$ & \\
\hline Taking some medicines & $12(20.7)$ & $14(16.3)$ & \\
\hline Nothing & $10(17.2)$ & $37(43)$ & \\
\hline Type of food consumed before chemotherapy & & & $0.003^{c}$ \\
\hline Carbohydrate & $8(16.3)$ & $37(49.3)$ & \\
\hline Dairy products & $20(40.8)$ & $21(28)$ & \\
\hline Vegetables & $9(18.4)$ & $7(9.3)$ & \\
\hline Protein & $10(20.4)$ & $6(8)$ & \\
\hline Fatty & $2(4.1)$ & $4(5.3)$ & \\
\hline Food consumption before the chemotherapy & $11(19)$ & $8(9.3)$ & $0.18^{c}$ \\
\hline The satisfaction of social support & $40(27.77)$ & $71(49.3)$ & $0.05^{\mathrm{c}}$ \\
\hline Having health insurance & $56(98.2)$ & $83(96.5)$ & $0.53^{c}$ \\
\hline Having history of smoking & $6(10.5)$ & $13(15.1)$ & $0.40^{\mathrm{c}}$ \\
\hline Having history of drug abuse & $5(5.8)$ & $6(10.5)$ & $0.30^{c}$ \\
\hline Having nausea expectation & $45(77.6)$ & $32(37.2)$ & $0.00^{c}$ \\
\hline
\end{tabular}

$\mathrm{a}$ Values are expressed as mean $\mathrm{SD}$ or No. (\%).

$\mathrm{b}$ Independent $t$-test.

Chi-square test. 


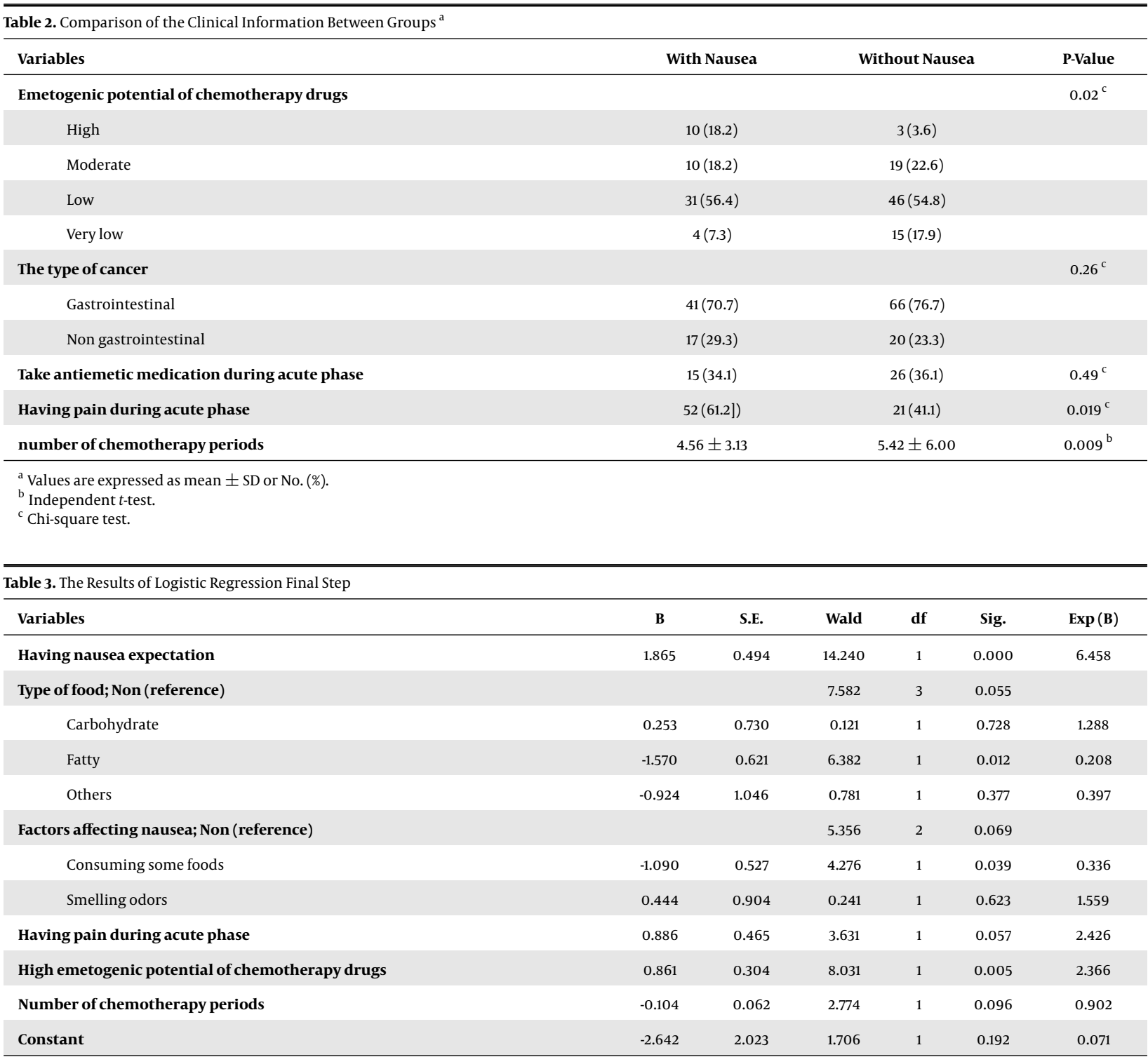

chemotherapy was 1.288 times higher than those who did not eat any food. However, Booth (2007) reported that lack of eating food before chemotherapy was a risk factor for nausea and vomiting (12).

Most patients with nausea reported having history of developing nausea at acute phase of chemotherapy following smelling a specific odor. Also, smelling a specific odor during the acute phase of chemotherapy led to 1.559-fold increase in the chance of nausea as compared to those who were not exposed to any odor. However, it is recommended to avoid using perfume and strong odors in delivering nursing care to patients under chemotherapy to prevent nausea. As far as the researchers of this study investigated, there is no study to examine the association be- tween smelling a specific odor and development of nausea; however, Soltani demonstrated that smelling odor caused pregnancy-induced nausea (17).

In the current study, expecting nausea was found to be significantly associated with development of nausea, such that the likelihood of nausea was 6.458 times higher in patients expecting nausea than in those not expecting nausea. Consistently, some studies have indicated that patients expecting nausea experience CIN more frequently (7, 14). Moreno et al. argued that having history of nausea and vomiting was a risk factor for nausea and vomiting after surgery (24), while Molassiotis et al. study showed that expecting nausea was not a significant factor for $\operatorname{CINV}(8)$. The findings of the current study highlight paying greater at- 
tention to the patients' expecting nausea and prevention of nausea via pharmacologic and non-pharmacologic approaches.

This study demonstrated that nausea and the type of chemotherapy drug were significantly associated with different emetogenic potential. Fraunholz et al. demonstrated that the emetogenic potential of chemotherapy protocol was the most important risk factor for the development of nausea and vomiting (10).

Our findings showed a significant association between feeling pain and developing CIN such that the likelihood of nausea was 2.426 times higher in patients feeling pain than in those without pain. Consistent with the current study, Gan and Watcha reported that postsurgical pain led to development of nausea and pain after surgery $(22,25)$. This study further confirms that greater attention should be paid to pain and preventing it in patients under chemotherapy using pharmacologic and nonpharmacologic approaches.

A strength of our study was that CIN-associated factors were investigated in patients with any type, rather than a specific type, of cancer referring for chemotherapy, including lymphoma, leukemia, lung cancer, breast cancer, larynx cancer, skin cancer, bone cancer, and brain cancer. In addition, this study investigated a wide spectrum of variables because nausea occurs most frequently at the acute phase of chemotherapy.

A limitation of the current study was that the results cannot be generalized to the late phase because we investigated the association of the above factors with CIN incidence only in the acute phase. Besides, because the patients were asked questions that required them to recall what happened 24 hours ago, they might not recall everything completely; and this increases the risk of recall bias.

\subsection{Conclusion}

The final model demonstrated that the likelihood of nausea was 2.426 and 6.458 times higher at the acute phase of chemotherapy in patients feeling pain and expecting nausea than in those without pain and not expecting nausea, respectively.

Chemotherapy consumption of carbohydrates or smelling a specific odor in the acute phase led to 1.288- and 1.559-fold increase in the chance of nausea, respectively, as compared to those who did not eat any food or were not exposed to any odor. The chance of nausea increases with the use of high nausea-inducing chemotherapy drugs. Therefore, nurses and physicians are recommended to pay greater attention to these factors and help to reduce CIN significantly through educating and mediating the factors.

\section{Acknowledgments}

The authors acknowledge the vice chancellor for Research and Technology of Hamadan University of Medical Sciences, Iran and all the patients who participated in the research.

\section{Footnotes}

Authors' Contribution: Zahra Khalili and Mahnaz Khatiban designed the study proposal and wrote the final article. Zahra Khalili and Mahnaz Moghadarikoosha collected the data. Mahdi Akbarzadeh, Zahra Khalili, Mahnaz Khatiban, and Maryam Seyedtabib analyzed the data.

Conflict of Interests: The authors received no funding and there is no conflict of interest to disclose.

Ethical Approval: This project was approved in the Ethics Committee of Hamadan University of Medical Sciences.

Funding/Support: This study was founded by the Vicechancellor for Research and Technology at Hamadan University of Medical Sciences (ID NO: 9208072463).

\section{References}

1. Jordan K, Gralla R, Jahn F, Molassiotis A. International antiemetic guidelines on chemotherapy induced nausea and vomiting (CINV): Content and implementation in daily routine practice. Eur J Pharmacol. 2014;722:197-202. doi: 10.1016/j.ejphar.2013.09.073. [PubMed: 24157984].

2. Aapro M, Molassiotis A, Dicato M, Pelaez I, Rodriguez-Lescure A, Pastorelli $\mathrm{D}$, et al. The effect of guideline-consistent antiemetic therapy on chemotherapy-induced nausea and vomiting (CINV): The Pan European Emesis Registry (PEER). Ann Oncol. 2012;23(8):1986-92. doi: 10.1093/annonc/mds021. [PubMed: 22396444].

3. Miller M, Kearney N. Chemotherapy-related nausea and vomiting past reflections, present practice and future management. Eur J Cancer Care (Engl). 2004;13(1):71-81. doi: 10.1111/j.1365-2354.2004.00446.x. [PubMed: 14961778].

4. Hesketh PJ. Chemotherapy-induced nausea and vomiting. $N$ Engl J Med. 2008;358(23):2482-94. doi: 10.1056/NEJMra0706547. [PubMed: 18525044].

5. Feyer P, Kleeberg UR, Steingraber M, Gunther W, Behrens M. Frequency of side effects in outpatient cancer care and their influence on patient satisfaction-a prospective survey using the PASQOC questionnaire. Support Care Cancer. 2008;16(6):567-75. doi: 10.1007/s00520008-0422-4. [PubMed: 18322707].

6. Henry DH, Viswanathan HN, Elkin EP, Traina S, Wade S, Cella D. Symptoms and treatment burden associated with cancer treatment: Results from a cross-sectional national survey in the U.S. Support Care Cancer. 2008;16(7):791-801. doi: 10.1007/s00520-007-0380-2. [PubMed: 18204940].

7. Roscoe JA, Morrow GR, Colagiuri B, Heckler CE, Pudlo BD, Colman L, et al. Insight in the prediction of chemotherapy-induced nausea. Support Care Cancer. 2010;18(7):869-76. doi: 10.1007/s00520-009-0723-2. [PubMed: 19701781]. [PubMed Central: PMC3017350].

8. Molassiotis A, Aapro M, Dicato M, Gascon P, Novoa SA, Isambert $\mathrm{N}$, et al. Evaluation of risk factors predicting chemotherapy-related 
nausea and vomiting: Results from a European prospective observational study. J Pain Symptom Manage. 2014;47(5):839-848 e4. doi: 10.1016/j.jpainsymman.2013.06.012. [PubMed: 24075401].

9. Hesketh PJ, Aapro M, Street JC, Carides AD. Evaluation of risk factors predictive of nausea and vomiting with current standard-ofcare antiemetic treatment: Analysis of two phase III trials of aprepitant in patients receiving cisplatin-based chemotherapy. Support Care Cancer. 2010;18(9):1171-7. doi: 10.1007/s00520-009-0737-9. [PubMed: 19756774].

10. Fraunholz I, Grau K, Weiss C, Rodel C. Patient- and treatment-related risk factors for nausea and emesis during concurrent chemoradiotherapy. Strahlenther Onkol. 2011;187(1):1-6. doi: 10.1007/s00066-0102196-0. [PubMed: 21234525].

11. Kim Y, Morrow GR. The effects of family support, anxiety, and posttreatment nausea on the development of anticipatory nausea: A latent growth model. J Pain Symptom Manage. 2007;34(3):265-76. doi: 10.1016/j.jpainsymman.2006.11.014. [PubMed: 17604591].

12. Booth CM, Clemons M, Dranitsaris G, Joy A, Young S, Callaghan W, et al. Chemotherapy-induced nausea and vomiting in breast cancer patients: A prospective observational study. J Support Oncol. 2007;5(8):374-80. [PubMed: 17944146].

13. Pirri C, Katris P, Trotter J, Bayliss E, Bennett R, Drummond P. Risk factors at pretreatment predicting treatment-induced nausea and vomiting in Australian cancer patients: A prospective, longitudinal, observational study. Support Care Cancer. 2011;19(10):1549-63. doi: 10.1007/s00520-010-0982-y. [PubMed: 20811914].

14. Colagiuri B, Zachariae R. Patient expectancy and post-chemotherapy nausea: A meta-analysis. Ann Behav Med. 2010;40(1):3-14. doi: 10.1007/s12160-010-9186-4. [PubMed: 20387022].

15. Nurane $\mathrm{S}$. [What makes nausea and vomiting worse during the first trimester of pregnancy]. Iran J Nurs Res. 2004;17(37):1-11. Persian.

16. Tabatabaei Chehr M, Mokhtari Far A. [Comparison of the incidence and severity of nausea and vomiting in pregnant women, housewives and workers attending prenatal care centers Bojnurd]. Med J Mashad Univ Med Sci. 2004;46(82):68-76. Persian.
17. Soltani A, Danesh Kajuri M, Safavi SH, Hosseini F. [Frequency and severity of nausea and vomiting in pregnancy and the related factors among pregnant women]. Iran J Nurs Res. 2007;19(48):95-102. Persian.

18. Kranke P, Apefel CC, Papenfuss T, Rauch S, Lobmann U, Rubsam B, et al. An increased body mass index is no risk factor for postoperative nausea and vomiting. A systematic review and results of original data. Acta Anaesthesiol Scand. 2001;45(2):160-6. [PubMed: 11167160].

19. Doubravska L, Dostalova K, Fritscherova S, Zapletalova J, Adamus M. Incidence of postoperative nausea and vomiting in patients at a university hospital. Where are we today? Biomed Pap Med Fac Univ Palacky Olomouc Czech Repub. 2010;154(1):69-76. doi: 10.5507/bp.2010.012. [PubMed: 20445713].

20. Apfel CC, Heidrich FM, Jukar-Rao S, Jalota L, Hornuss C, Whelan RP, et al. Evidence-based analysis of risk factors for postoperative nausea and vomiting. BrJAnaesth. 2012;109(5):742-53. doi:10.1093/bja/aes276. [PubMed: 23035051].

21. Leslie K, Myles PS, Chan MT, Paech MJ, Peyton P, Forbes A, et al. Risk factors for severe postoperative nausea and vomiting in a randomized trial of nitrous oxide-based vs nitrous oxide-free anaesthesia. Br J Anaesth. 2008;101(4):498-505. doi: 10.1093/bja/aen230. [PubMed: 18682411].

22. Gan TJ. Risk factors for postoperative nausea and vomiting. Anesth Analg. 2006;102(6):1884-98. doi: 10.1213/01.ANE.0000219597.16143.4D. [PubMed: 16717343].

23. Laugsand EA, Fladvad T, Skorpen F, Maltoni M, Kaasa S, Fayers P, et al Clinical and genetic factors associated with nausea and vomiting in cancer patients receiving opioids. EurJ Cancer. 2011;47(11):1682-91. doi: 10.1016/j.ejca.2011.04.014. [PubMed: 21570824].

24. Moreno C, Veiga D, Pereira H, Martinho C, Abelha F. Postoperative nausea and vomiting: Incidence, characteristics and risk factors-a prospective cohort study. Rev Esp Anestesiol Reanim. 2013;60(5):24956. doi: 10.1016/j.redar.2013.02.005. [PubMed: 23582584].

25. Watcha MF, White PF. Postoperative nausea and vomiting. Its etiology, treatment, and prevention. Anesthesiology.1992;77(1):162-84. doi: 10.1097/00000542-199207000-00023. [PubMed:1609990]. 